\title{
Weakly invariant subspaces for multivalued linear operators on Banach spaces
}

\author{
Gerald Wanjala \\ Department of Mathematics and Statistics, Sultan Qaboos University, P. O. Box 36, PC 123, Al Khoud, Sultanate of Oman.
}

Communicated by $\mathrm{Y}$. Hu

\begin{abstract}
Peter Saveliev generalized Lomonosov's invariant subspace theorem to the case of linear relations. In particular, he proved that if $\mathcal{S}$ and $\mathcal{T}$ are linear relations defined on a Banach space $X$ and having finite dimensional multivalued parts and if $\mathcal{T}$ right commutes with $\mathcal{S}$, that is, $\mathcal{S} \mathcal{T} \subset \mathcal{T} \mathcal{S}$, and if $\mathcal{S}$ is compact then $\mathcal{T}$ has a nontrivial weakly invariant subspace. However, the case of left commutativity remained open. In this paper, we develop some operator representation techniques for linear relations and use them to solve the left commutativity case mentioned above under the assumption that $\mathcal{S} \mathcal{T}(0)=\mathcal{S}(0)$ and $\mathcal{T} \mathcal{S}(0)=\mathcal{T}(0)$.
\end{abstract}

Keywords: Linear relations, weakly invariant subspaces.

2010 MSC: 47A06, 47A15.

(C) 2018 All rights reserved.

\section{Introduction}

Let $X$ be normed linear space and $T$ a bounded linear operator on $X$. A closed subspace $\mathcal{M}$ of $X$ is said to be a nontrivial invariant subspace for $\mathrm{T}$ if $\{0\} \neq \mathcal{M} \neq \mathrm{X}$ and $\mathrm{T} x \in \mathcal{M}$ for every $x \in \mathcal{M}$. The invariant subspace problem is the question whether every bounded operator on $X$ has a nontrivial closed invariant subspace. One of the oldest results in the theory of invariant subspaces is the theorem of Aronszajn and Smith [1], published in 1954, that every compact operator on a Banach space $X$ has a nontrivial invariant subspace. A much stronger result and probably one of the landmark theorems in the area of invariant subspaces was given in 1973 by Lomonosov [6], a special case of which states that:

If an operator $T$ on a Banach space commutes with a non-zero compact operator, then $T$ has a nontrivial invariant subspace.

Using fixed point techniques for multivalued operators, Saveliev [8] generalized Lomonosov's result to linear relations with finite dimensional multivalued parts which right commute with a compact linear relation but the case of left commutativity remained open. In this paper, we employ operator representations of linear relations with finite dimensional multivalued parts to solve a case of left commutativity. By a linear relation here we mean a multivalued operator $\mathcal{T}: D(\mathcal{T}) \subset X \rightarrow Y$ between linear spaces such that

Email address: gwanjala@squ.edu.om; wanjalag@yahoo.com (Gerald Wanjala)

doi: $10.22436 /$ jnsa.011.07.01

Received: 2017-08-30 Revised: 2017-12-14 Accepted: 2018-04-18 
1. $\quad \mathcal{T}(x+y)=\mathcal{T}(x)+\mathcal{T}(y)$,

2. $\mathcal{T}(\alpha x)=\alpha \mathcal{T}(x)$,

for all $x, y \in D(\mathcal{T})$, the domain of $\mathcal{T}$, and any nonzero scalar $\alpha$. Here, addition on the righthand side of (1) above should be understood to be addition of sets. If $D(\mathcal{T})=X$, we denoted by $\operatorname{LR}(X, Y)$ the class of all linear relations from $X$ into $Y$ and we denote $\operatorname{LR}(X, X)$ by $\operatorname{LR}(X)$. We say that $T$ is a linear relation on $X$ if $T \in \operatorname{LR}(X)$.

Let $\mathcal{S}$ and $\mathcal{T}$ be two linear relations on a linear space $X$ and let $G(\mathcal{S})$ denote the graph of $\mathcal{S}$. We say that $\mathcal{S}$ left commutes with $\mathcal{T}$ if

$$
\mathrm{G}(\mathcal{S T}) \subset \mathrm{G}(\mathcal{T S})
$$

We write $\mathcal{S} \mathcal{T} \subset \mathcal{T} \mathcal{S}$ to mean that $\mathcal{S}$ left commutes with $\mathcal{T}$. Right commutativity is defined in a similar way. Recall that

$$
\mathrm{D}(\mathcal{S T})=\{x \in \mathrm{D}(\mathcal{T}): \mathcal{T}(x) \in \mathrm{D}(\mathcal{S})\}
$$

For a detailed account of the theory of linear relations we refer to [3].

For purposes of completeness and for the convenience of the reader, we conclude this section by recalling some auxiliary results on quotient spaces and complementary subspaces. Let $M$ and $N$ be subspaces of a linear space $X$. We define the sum $M+N$ of $M$ and $N$ to be

$$
M+N=\{m+n:(m, n) \in M \times N\} .
$$

If $M$ is a subspace of a linear space $X$ then $X / M$ denotes the linear space of all equivalent classes $[x]=$ $x+M$, where $x$ is equivalent to $y$ if and only if $x-y \in M$, with $x, y \in X$. The following two lemmas, together with their proofs can be found in $[2,7]$.

Lemma 1.1. Let $\mathrm{X}$ be a normed space, $\mathrm{E}$ a subspace of $\mathrm{X}$ and $\mathrm{F}$ a subspace of $\mathrm{E}$.

(a) If $\mathrm{E}$ is a complemented subspace of $\mathrm{X}$ and $\mathrm{F}$ is a complemented subspace of $\mathrm{E}$ then $\mathrm{F}$ is a complemented subspace of $X$.

(b) If $\mathrm{F}$ is a complemented subspace of $\mathrm{X}$, then it is a complemented subspace of $\mathrm{E}$.

Lemma 1.2. Let $X$ be a normed linear space and let $\mathrm{M}$ and $\mathrm{N}$ be topologically complemented subspaces of $\mathrm{X}$ and let

$$
\pi: X \rightarrow X / M
$$

be the natural quotient map. Then $\left.\pi\right|_{N}$, the restriction of $\pi$ to $\mathrm{N}$ is a homeomorphism.

\section{Some properties of linear relations}

From now on, $\mathrm{X}$ and $\mathrm{Y}$ will denote normed spaces unless stated otherwise.

Definition 2.1. Let $\mathcal{T}: X \rightarrow Y$ be a linear relation. The inverse of $\mathcal{T}$ is the linear relation $\mathcal{T}^{-1}$ defined by $\mathcal{T}^{-1}:=\{(y, x) \in Y \times X:(x, y) \in G(\mathcal{T})\}$.

The fact that $\mathcal{T}$ is linear if and only if

$$
\mathcal{T}(\alpha x+\beta y)=\alpha \mathcal{T}(x)+\beta \mathcal{T}(y)
$$

implies that both $\mathcal{T}(0)$ and $\mathcal{T}^{-1}(0)$ are linear subspaces. See [3] for the following lemma.

Lemma 2.2. Let $\mathcal{T} \in \operatorname{LR}(X, Y)$ and let $x \in \mathrm{D}(\mathcal{T})$. Then

$$
\mathcal{T}(x)=y+T(0)
$$

for every $y \in T(x)$. 
Definition 2.3. Let $\mathcal{T}: X \rightarrow Y$ be a linear relation. Then

1. $\mathcal{T}(M)=\bigcup\{\mathcal{T}(x) \mid x \in M\}$ for each $M \subset X$,

2. $\mathcal{T}^{-1}(\mathrm{~N})=\{x \in X \mid \mathcal{T}(\mathrm{x}) \cap \mathrm{N} \neq \emptyset\}$ for each $\mathrm{N} \subset \mathrm{Y}$.

The definition of continuity given below takes into consideration the fact that there are linear relations $\mathcal{T}: X \rightarrow Y$ from a topological space $X$ to a topological space $Y$ for which $\mathcal{T}^{-1}(U)$ is open for every open $U$ in $Y$ but $\mathcal{T}^{-1}(B)$ is not closed for some closed $B$ in $Y$. In other words, the various equivalent definitions of continuity of single valued functions have to be considered separately in the case of linear relations.

Definition 2.4. Let $\mathcal{T}: X \rightarrow Y$ be a linear relation. Then we say that

(a) $\mathcal{T}$ is upper semi-continuous if $\mathcal{T}^{-1}(\mathrm{~B})$ is closed for every closed $\mathrm{B} \in \mathrm{Y}$,

(b) $\mathcal{T}$ is lower semi-continuous if $\mathcal{T}^{-1}(\mathrm{U})$ is open for every open $U \in Y$,

(c) $\mathcal{T}$ is continuous if it is both upper semi-continuous and lower semi-continuous.

We will concentrate on the classes $\mathrm{LR}_{0}(\mathrm{X})$ and $\mathrm{LR}_{0}(\mathrm{X}, \mathrm{Y})$ where

$$
\operatorname{LR}_{0}(X, Y)=\{\mathcal{T} \in \operatorname{LR}(X, Y): \mathcal{T} \text { is continuous, } \operatorname{dim} \mathcal{T}(0)<\infty\}
$$

and $\mathrm{LR}_{0}(X)=\mathrm{LR}_{0}(X, X)$. Note that $\mathrm{B}(\mathrm{X}, \mathrm{Y}) \subset \mathrm{LR}_{0}(\mathrm{X}, \mathrm{Y})$, where $\mathrm{B}(\mathrm{X}, \mathrm{Y})$ denotes the class of all bounded linear operators from $X$ into $Y$. See [8] for the following lemma.

Lemma 2.5. If $\mathcal{S} \in \mathrm{LR}_{0}(\mathrm{X}, \mathrm{Z})$ and $\mathcal{T} \in \mathrm{LR}_{0}(\mathrm{Z}, \mathrm{Y})$, then $\mathcal{T S} \in \mathrm{LR}_{0}(\mathrm{X}, \mathrm{Y})$.

For $\mathcal{T} \in \operatorname{LR}_{0}(X, Y)$, let $Q_{\mathcal{T}}$ denote the natural quotient map with domain $Y$ and null space $\mathcal{T}(0)$. Then $\mathrm{Q}_{\mathcal{T}} \mathcal{T}$ is a single valued linear operator [3, p. 25].

Definition 2.6. Let $\mathcal{T} \in \mathrm{LR}_{0}(X, Y)$. $\mathcal{T}$ is said to be compact if the single valued operator $\mathrm{Q}_{\mathcal{T}} \mathcal{T}$ is compact.

\section{Operator representation of continuous linear relations}

Let $\mathcal{T} \in \mathrm{LR}_{0}(\mathrm{X}, \mathrm{Y})$. In this section, we use the fact that $\mathcal{T}(0)$ is topologically complemented in $Y$ with topological complement $\mathcal{T}(0)^{\mathrm{c}}$ to show the existence of a linear operator $A: X \rightarrow \mathcal{T}(0)^{\mathrm{c}} \subset Y$ such that

$$
\mathcal{T}(x)=\mathcal{T}(0)+A x
$$

for every $x \in X=D(\mathcal{T})$. We employ the techniques developed in [9] where a similar result was proved for sectorial linear relations on Hilbert spaces. This result is well known for linear relations $\mathcal{T}$ on Hilbert spaces for which $G(\mathcal{T})$, the graph of $\mathcal{T}$, is closed. The fact that the range of $A$ is contained in $\mathcal{T}(0)^{c}$, the topological complement of $\mathcal{T}(0)$ in $Y$ is very crucial in our subsequent proofs. We refer to (3.1) as an operator representation of the linear relation $\mathcal{T}$ with operator part $A$.

Theorem 3.1. Let $X$ and $Y$ be Banach spaces and let $\mathcal{T} \in \operatorname{LR}(X, Y)$. If $\mathcal{T}(0)$ is topologically complemented in $Y$ with topological complement $\mathcal{T}(0)^{\mathrm{c}}$ then there exits a linear operator $\mathrm{A}: \mathrm{X} \rightarrow \mathcal{T}(0)^{\mathrm{c}}$ such that

$$
\mathcal{T}(x)=T(0)+A x \quad \text { for all } x \in X .
$$

Proof. Let $\mathcal{T}(0)^{\mathrm{c}}$ be a topological compliment of $\mathcal{T}(0)$ in $Y$ and let $x \in X$. Then every $y \in \mathcal{T}(x)$ can be uniquely decomposed as

$$
y=z+w, \quad z \in \mathcal{T}(0)^{\mathrm{c}}, w \in \mathcal{T}(0) .
$$

Lemma (2.2) implies that

$$
\mathcal{T}(x)=y+\mathcal{T}(0)=z+[w+\mathcal{T}(0)]=z+\mathcal{T}(0) .
$$


Let $\mathrm{P}$ be the projection of $X$ onto $\mathcal{T}(0)^{\mathrm{c}}$ along $\mathcal{T}(0)$. Equality (3.3) shows that Py is irrespective of the choice of $y \in \mathcal{T}(x)$, that is, $P y_{1}=P y_{2}$ for every $y_{1}, y_{2} \in \mathcal{T}(x)$. Define $A: X \rightarrow \mathcal{T}(0)^{c}$ by

$$
A x=P y, \quad y \in \mathcal{T}(x) .
$$

Then $A$ is a well defined linear operator whose linearity follows from that of $P$ and $\mathcal{T}$.

Note that every $\mathcal{T} \in \mathrm{LR}_{0}(\mathrm{X})$ admits representation (3.2) since $\operatorname{dim} \mathcal{T}(0)<\infty$ and every finite dimensional subspace of a Banach space is topologically complemented.

Lemma 3.2. Let $\mathcal{T} \in \mathrm{LR}_{0}(\mathrm{X})$ and let

$$
\mathcal{T}(x)=\mathcal{T}(0)+A(x)
$$

be an operator representation of $\mathcal{T}$. Then

$$
\mathrm{G}(\mathcal{T})=\mathrm{G}(A) \oplus(\{0\} \times \mathcal{T}(0)) .
$$

If $\mathrm{P}$ is the projection of $\mathrm{G}(\mathcal{T})$ onto $\mathrm{G}(\mathrm{A})$ along $\{0\} \times \mathcal{T}(0)$, then

$$
P(x, y)=(x, A x)
$$

for every $(x, y) \in G(\mathcal{T})$.

Proof. Equality (3.5) is a consequence of (3.4). Let $y \in \mathcal{T}(x)$. We see from (3.2) that $y=z+A(x)$ for some $z \in \mathcal{T}(0)$ and so $(x, y)=(x, A x)+(0, z)$. Hence

$$
P(x, y)=(x, A x)
$$

The following lemma enables us to extend Aronszajn Smith's theorem [1] which states that every compact operator on a Banach space $X$ has a nontrivial invariant subspace to the case of compact linear relations $\mathcal{T} \in \mathrm{LR}_{0}(\mathrm{X})$.

Lemma 3.3. Let $\mathcal{T}(x)=\mathcal{T}(0)+A x$ be an operator representation of $\mathcal{T} \in \mathrm{LR}_{0}(\mathrm{X})$. Then $\mathcal{T}$ is compact if and only if the operator $\mathrm{A}$ is compact.

Proof. Let $\mathcal{T}(0)^{\mathrm{c}}$ be a topological complement of $\mathcal{T}(0)$ in $X, Z=X / \mathcal{T}(0)$ and consider the natural quotient mapping $\pi$ with domain $X$ and kernel $\mathcal{T}(0)$. Denote by $\widehat{\pi}$ the restriction of $\pi$ to $\mathcal{T}(0)^{\mathrm{c}}$. Lemma 1.2 implies that the mapping $\widehat{\pi}: \mathcal{T}(0)^{\mathfrak{c}} \rightarrow Z$ is a homeomorphism. Since

$$
\mathrm{Q}_{\mathcal{T}} \mathcal{T}(x)=\mathrm{Q}_{\mathcal{T}}(\mathcal{T}(0)+\mathrm{A}(\mathrm{x}))
$$

for all $x \in X$, the linearity of $Q_{\mathcal{T}}$ implies that

$$
\mathrm{Q}_{\mathcal{T}} \mathcal{T}(x)=\mathrm{Q}_{\mathcal{T}}(\mathcal{T}(0))+\mathrm{Q}_{\mathcal{T}} \mathrm{A}(\mathrm{x})=\mathrm{Q}_{\mathcal{T}} \mathrm{A} x=[A x],
$$

where $[A(x)]$ denotes the equivalent class of $A(x)$ is Z. From (3.7) we see that for $x \in X$,

$$
\mathrm{Q}_{\mathcal{T}} \mathcal{T}(x)=\widehat{\pi} \mathrm{A}(x)
$$

and that

$$
A(x)=(\widehat{\pi})^{-1} Q_{\mathcal{T}} \mathcal{T}(x) .
$$

The lemma then follows from (3.8) and (3.9) and the fact that the composition of a compact linear operator and a bounded linear operator yields a compact linear operator [5]. 


\section{Operator Representation of Compositions of Linear Relations}

In this section, we consider an operator representation $A_{\mathcal{S T}}$ of the composition $\mathcal{S T}$ of linear relations $\mathcal{S}$ and $\mathcal{T}$ in $\operatorname{LR}_{0}(X)$. We establish a relationship between operator parts of the individual linear relations $\mathcal{S}$ and $\mathcal{T}$ and that of their composition $\mathcal{S} \mathcal{T}$. In particular, we show that if $A_{\mathcal{S T}}$ is an operator part of the composition $\mathcal{S} \mathcal{T}$ then it is a composition of operator part of $\mathcal{S}$ and $\mathcal{T}$ in that order if $\mathcal{S T}(0)=\mathcal{S}(0)$. We then use this result to show that if $\mathcal{T}$ right (left) commutes with $\mathcal{S}$ and if $\mathcal{S} \mathcal{T}(0)=\mathcal{S}(0)$ and $\mathcal{T} \mathcal{S}(0)=\mathcal{T}(0)$ then their operator parts also commute. This last result is used in the next section to prove the existence of a nontrivial weakly invariant subspace for $\mathcal{S}$ under some assumptions on $\mathcal{T}$.

Lemma 4.1. Let $\mathrm{X}$ be a Banach space and consider $\mathcal{S}, \mathcal{T} \in \mathrm{LR}_{0}(\mathrm{X})$ with operator representations

$$
\mathcal{S}(x)=\mathcal{S}(0)+A_{\mathcal{S}}(x) \text { and } \mathcal{T}(x)=\mathcal{T}(0)+A_{\mathcal{T}}(x)
$$

and let

$$
\mathcal{S T}(x)=\mathcal{S T}(0)+A_{\mathcal{S T}}(x)
$$

be an operator representation of $\mathcal{S} \mathcal{T}$. Denote by $\mathrm{P}_{\mathcal{S T}}$ the projection of $\mathrm{X}$ onto $\mathcal{S} \mathcal{T}(0)^{\mathrm{c}}$ along $\mathcal{S T}(0)$. Then for all $\mathrm{x} \in \mathrm{X}$,

(a) $A_{\mathcal{S T}}(x)=\mathrm{P}_{\mathcal{S} \mathcal{T}} A_{\mathcal{S}} A_{\mathcal{T}}(x)$ and,

(b) if $\mathcal{S}(0)=\mathcal{S} \mathcal{T}(0)$ then $A_{\mathcal{S}} A_{\mathcal{T}}(x)=A_{\mathcal{S T}}(x)$.

Proof. Let $x \in X$. Then

$$
\begin{aligned}
\mathcal{S T}(x) & =\mathcal{S}(\mathcal{T}(x)) \\
& =\mathcal{S}\left(\mathcal{T}(0)+A_{\mathcal{T}}(x)\right) \\
& =\mathcal{S}(\mathcal{T}(0))+\mathcal{S}\left(A_{\mathcal{T}}(x)\right) \\
& =S \mathcal{T}(0)+\mathcal{S}(0)+A_{\mathcal{S}}\left(A_{\mathcal{T}}(x)\right) \\
& =\mathcal{S T}(0)+A_{\mathcal{S}} A_{\mathcal{T}}(x) .
\end{aligned}
$$

Hence

$$
\mathcal{S T}(x)=\mathcal{S T}(0)+A_{\mathcal{S}} A_{\mathcal{T}}(x)
$$

for all $x \in X$. Recall that $\mathcal{S T}$ has an operator representation

$$
\mathcal{S T}(x)=\mathcal{S T}(0)+A_{\mathcal{S T}}(x),
$$

and therefore

$$
\mathcal{S T}(0)+A_{\mathcal{S T}}(x)=S \mathcal{S}(0)+A_{\mathcal{S}} A_{\mathcal{T}}(x) .
$$

Since $A_{\mathcal{S} \mathcal{T}}(x) \in \mathcal{S T}(0)^{\mathfrak{c}}$, application of $\mathrm{P}_{\mathcal{S} \mathcal{T}}$ on both sides of (4.3) shows that

$$
A_{\mathcal{S T}}(x)=\mathrm{P}_{\mathcal{S} \mathcal{T}} A_{\mathcal{S}} A_{\mathcal{T}}(x) .
$$

This proves part (a) of the lemma. Part (b) follows from the fact that $A_{\mathcal{S}}(x) \in \mathcal{S}(0)^{\mathfrak{c}}$ and that $\mathcal{S}(0)=$ $\mathcal{S T}(0)$.

Below we give an example of linear relations $\mathcal{S}$ and $\mathcal{T}$ for which $\mathcal{S}(0)=\mathcal{S T}(0)$ and $\mathcal{T}(0)=\mathcal{T} \mathcal{S}(0)$.

Example 4.2. Let $X$ be a Hilbert space and consider the Hilbert space $\widetilde{\mathcal{H}}=\mathcal{H} \oplus \mathcal{H}$ and let $u$ and $v$ be arbitrary but fixed elements of $\mathcal{H}$. If $\mathcal{S}$ and $\mathcal{T}$ are linear relations whose graphs are defined by

$$
G(\mathcal{S})=\left(\left(\begin{array}{c}
x \\
y
\end{array}\right),\left(\begin{array}{c}
x \\
\alpha u
\end{array}\right), \alpha \in \mathbb{C}\right), G(\mathcal{T})=\left(\left(\begin{array}{l}
x \\
y
\end{array}\right),\left(\begin{array}{c}
x \\
\beta u+\gamma \nu
\end{array}\right), \beta, \gamma \in \mathbb{C}\right)
$$

then $\mathcal{S}(0)=\mathcal{S} \mathcal{T}(0)$ and $\mathcal{T}(0)=\mathcal{T} \mathcal{S}(0)$. 
The following theorem establishes the commutativity of operator parts of linear relations $\mathcal{S}$ and $\mathcal{T}$.

Theorem 4.3. Let $X$ be a Banach space and let $\mathcal{S}(x)=\mathcal{S}(0)+A_{\mathcal{S}}(x)$ and $\mathcal{T}(x)=\mathcal{T}(0)+A_{\mathcal{T}}(x)$ be operator representations of linear relations $\mathcal{S}$ and $\mathcal{T}$ in $\mathrm{LR}_{0}(\mathrm{X})$. If $\mathcal{T}$ right commutes with $\mathcal{S}$, that is, $\mathcal{S} \mathcal{T} \subset \mathcal{T} \mathcal{S}$, and if $\mathcal{S}(0)=\mathcal{S} \mathcal{T}(0)$ and $\mathcal{T}(0)=\mathcal{T} \mathcal{S}(0)$ then the operators $A_{\mathcal{S}}$ and $A_{\mathcal{T}}$ commute, that is,

$$
A_{\mathcal{S}} A_{\mathcal{T}}=A_{\mathcal{T}} A_{\mathcal{S}}
$$

Proof. Since $\mathcal{S}, \mathcal{T} \in \mathrm{LR}_{0}(\mathrm{X})$, it follows that $\mathcal{S} \mathcal{T} \in \mathrm{LR}_{0}(\mathrm{X})$ and $\mathcal{T} \mathcal{S} \in \mathrm{LR}_{0}(\mathrm{X})$. Lemma 4.1 implies that $\mathcal{S} \mathcal{T}$ and $\mathcal{T S}$ have operator representations $\mathcal{S} \mathcal{T}(x)=\mathcal{S} \mathcal{T}(0)+A_{\mathcal{S}} A_{\mathcal{T}}(x)$, and $\mathcal{T} \mathcal{S}(x)=\mathcal{T} \mathcal{S}(0)+A_{\mathcal{T}} A_{\mathcal{S}}(x)$ for all $x \in X$. Since $\mathcal{S} \mathcal{T} \subset \mathcal{T} \mathcal{S}$, we see that for all $x \in X$,

$$
\mathcal{S} \mathcal{T}(0)+A_{\mathcal{S}} A_{\mathcal{T}}(x) \subset \mathcal{T} \mathcal{S}(0)+A_{\mathcal{T}} A_{\mathcal{S}}(x) .
$$

In particular, $x=0$ yields

$$
\mathcal{S T}(0) \subset \mathcal{T S}(0) .
$$

Relation (4.4) means that for every $x \in X$, there exists a $z \in \mathcal{T S}(0)$ such that

$$
A_{\mathcal{S}} A_{\mathcal{T}}(x)=z+A_{\mathcal{T}} A_{\mathcal{S}}(x)
$$

and so

$$
A_{\mathcal{S}} A_{\mathcal{T}}(x)-A_{\mathcal{T}} A_{\mathcal{S}}(x)=z \in \mathcal{T} \mathcal{S}(0) .
$$

Consider the quotient space $X / \mathcal{T S}(0)$ and for $y \in X$, let $[y]_{X / \mathcal{T S}(0)}$ denote the quotient class of $y$ in $X / \mathcal{T S}(0)$. Then we see from (4.6) that for all $x \in X$,

$$
\left[A_{\mathcal{T}} A_{\mathcal{S}}(x)\right]_{X / \mathcal{T S}(0)}=\left[A_{\mathcal{S}} A_{\mathcal{T}}(x)\right]_{X / \mathcal{T S}(0)} .
$$

Let $x \in X$ and consider the decompositions

$$
x=y_{1}+y_{2} \in \mathcal{S T}(0) \oplus \mathcal{S T}(0)^{c}=X
$$

and

$$
x=z_{1}+z_{2} \in \mathcal{T S}(0) \oplus \mathcal{T S}(0)^{\mathrm{c}}=\mathrm{X} .
$$

Lemma 1.2 implies that the mappings

$$
\Pi: X / \mathcal{S T}(0) \oplus \mathcal{S T}(0) \rightarrow X ; \quad \Pi\left([x]_{X / \mathcal{S}} \mathcal{T}(0)+y_{1}\right)=x
$$

and

$$
\pi: X \rightarrow X / \mathcal{T S}(0) \oplus \mathcal{T S}(0) ; \quad \pi(x)=\left([x]_{X / \mathcal{T S}(0}+z_{1}\right)
$$

are both bijective. This observation implies that the mapping

$$
\widehat{\Pi}:=\pi \circ \Pi: X / \mathcal{S T}(0) \oplus \mathcal{S} \mathcal{T}(0) \rightarrow X / \mathcal{T S}(0) \oplus \mathcal{T S}(0),
$$

which is defined by

$$
\widehat{\Pi}\left([x]_{X / \mathcal{S} \mathcal{T}(0)}+y_{1}\right)=\left([x]_{X / \mathcal{T} \mathcal{S}(0)}+z_{1}\right)
$$

is also bijective. For the element $A_{\mathcal{S}} A_{\mathcal{T}}(x) \in \mathcal{S} \mathcal{T}(0)^{\mathrm{c}}$, (4.5) implies that

$$
\begin{aligned}
\widehat{\Pi}\left(\left[A_{\mathcal{S}} A_{\mathcal{T}}(x)\right]_{\mathrm{X} / \mathcal{S} \mathcal{T}(0)}+0\right) & =\left(\left[A_{\mathcal{S}} A_{\mathcal{T}}(x)\right]_{\mathrm{X} / \mathcal{T S}(0)}+z\right) \\
& =\left(\left[A_{\mathcal{T}} A_{\mathcal{S}}(x)\right]_{\mathrm{X} / \mathcal{T S}(0)}+z\right),
\end{aligned}
$$

where the last equality in (4.8) follows from (4.7). Hence if $z$ is as given by (4.7), then

$$
\widehat{\Pi}\left(\left[A_{\mathcal{S}} A_{\mathcal{T}}(x)\right]_{X / \mathcal{S} \mathcal{T}(0)}\right)=\left[A_{\mathcal{T}} A_{\mathcal{S}}(x)\right]_{X / \mathcal{T} \mathcal{S}(0)}+z .
$$


Consider the decomposition

$$
w=u+v \in S \mathcal{S T}(0) \oplus \mathcal{S T}(0)^{\mathfrak{c}}=\mathrm{X}
$$

of the element $w=A_{\mathcal{T}} A_{\mathcal{S}}(x)+z \in \mathcal{T S}(0) \oplus \mathcal{T S}(0)^{\mathfrak{c}}=X$. Then

$$
\widehat{\Pi}^{-1}\left(\left[A_{\mathcal{T}} A_{\mathcal{S}}(x)\right]_{\mathrm{X} / \mathcal{T} \mathcal{S}(0)}+z\right)=\left(\left[A_{\mathcal{T}} A_{\mathcal{S}}(x)\right]_{\mathrm{X} / \mathcal{S} \mathcal{T}(0)}+u\right) .
$$

Since $\widehat{\Pi}$ is bijective, (4.9) and (4.10) imply that

$$
\left[A_{\mathcal{T}} A_{\mathcal{S}}(x)\right]_{\mathrm{X} / \mathcal{S} \mathcal{T}(0)}+u=\left[A_{\mathcal{S}} A_{\mathcal{T}}(x)\right]_{\mathrm{X} / \mathcal{S T}(0)} .
$$

Let $\mathrm{P}$ be the projection of $\mathrm{X} / \mathcal{S T}(0) \oplus \mathcal{S T}(0)$ onto $\mathrm{X} / \mathcal{S T}(0)$ along $\mathcal{S T}(0)$. Applying $\mathrm{P}$ to both side of the equality (4.11) shows that

$$
\left[A_{\mathcal{T}} A_{\mathcal{S}}(x)\right]_{X / \mathcal{S T}(0)}=\left[A_{\mathcal{S}} A_{\mathcal{T}}(x)\right]_{X / \mathcal{S T}(0)}
$$

for all $x \in X$. This equality implies that

$$
A_{\mathcal{T}} A_{\mathcal{S}}(x)-A_{\mathcal{S}} A_{\mathcal{T}}(x) \in \mathcal{S T}(0) .
$$

From (4.6) and (4.12) we see that there exists an elements $s=-z \in \mathcal{S T}(0)$ such that

$$
A_{\mathcal{T}} A_{\mathcal{S}}(x)=s+A_{\mathcal{S}} A_{\mathcal{T}}(x) .
$$

Let $\mathrm{Q}$ be the projection of $X$ onto $\mathcal{S T}(0)^{\mathfrak{c}}$ along $\mathcal{S T}(0)$. Since $\mathcal{T S}(0)^{\mathfrak{c}} \subset \mathcal{S T}(0)^{\mathrm{c}}$, application of $\mathrm{Q}$ on both sides of (4.13) yields the required result

$$
A_{\mathcal{T}} A_{\mathcal{S}}(x)=A_{\mathcal{S}} A_{\mathcal{T}}(x) .
$$

Note that we have used the fact that $\mathcal{S}(0)=\mathcal{S T}(0)$ and $\mathcal{T}(0)=\mathcal{T S}(0)$ in the application of the projection $\mathrm{Q}$ in (4.13).

\section{Weakly Invariant Subspaces}

Let $X$ be a normed linear space and let $\mathcal{T} \in \operatorname{LR}(X)$. A subspace $M$ of $X$ is said to be a weakly invariant subspace for $\mathcal{T}$ or a $\mathcal{T}$-weakly invariant subspace if $\mathcal{T}(M) \cap M \neq \emptyset$. For any $\mathcal{T} \in \operatorname{LR}(X)$, the subspace $\mathcal{T}(0)$ is trivially $\mathcal{T}$-weakly invariant. This follows from Lemma 2.2 since $\mathcal{T}(0)$ is a linear subspace and therefore $0 \in \mathcal{T}(0)$. The invariant subspace for linear relations $\mathcal{T} \in \operatorname{LR}(X)$ therefore is the question of existence of weakly invariant subspaces $M$ such that $\{0\} \neq M \neq X$ and $M \neq \mathcal{T}(0)$. We begin with the following simple result.

Lemma 5.1. Let $\mathcal{T}$ be a linear relation in $\mathrm{LR}_{0}(\mathrm{X})$ with operator representation $\mathcal{T}(x)=\mathcal{T}(0)+A x$. If $A$ has a nontrivial invariant subspace then $\mathcal{T}$ has a nontrivial weakly invariant subspace.

Proof. Let $M$ be a nontrivial invariant subspace for $A$. Since $R(A) \subset \mathcal{T}(0)^{c}$, it follows that $M \neq \mathcal{T}(0)$. The subspace $M$ is a weakly invariant subspace for $\mathcal{T}$ since $A x \in \mathcal{T}(x)=\mathcal{T}(0)+A x$ and so $\mathcal{T}(x) \cap M \neq \emptyset$.

Next we consider the following linear relation form of the Aronszajn Smith's theorem [1].

Theorem 5.2. Let $\mathcal{T} \in \mathrm{LR}_{0}(\mathrm{X})$ be compact. Then $\mathcal{T}$ has a nontrivial weakly invariant subspace.

Proof. Let $\mathcal{T}(x)=\mathcal{T}(0)+A x$ be the operator representation of $\mathcal{T}$. Since $\mathcal{T}$ is compact, it follows from Lemma 3.3 that $A$ is a compact operator and therefore has a nontrivial invariant subspace $M$. The result then follows from Lemma 5.1.

We are now in a position to prove the existence of a nontrivial weakly invariant subspace for a linear relation $\mathcal{S} \in \mathrm{LR}_{0}(\mathrm{X})$ that left commutes with a compact linear relation $\mathcal{T}$ and satisfies the conditions of Theorem 4.3. 
Theorem 5.3. Let $X$ be a Banach space and let $\mathcal{S}, \mathcal{T} \in \mathrm{LR}_{0}(\mathrm{X})$. Suppose further that $\mathcal{S}$ left commutes with $\mathcal{T}$, that is, $\mathcal{S T} \subset \mathcal{T S}$, and that $\mathcal{S}(0)=\mathcal{S} \mathcal{T}(0)$ and $\mathcal{T}(0)=\mathcal{T} \mathcal{S}(0)$. If $\mathcal{T}$ is compact then there exists a nontrivial weakly invariant subspace $\mathrm{M} \subset \mathrm{X}$ for $\mathcal{S}$.

Proof. Let $\mathcal{S}, \mathcal{T} \in \mathrm{LR}_{0}(\mathrm{X})$ with operator representations $\mathcal{S}(x)=\mathcal{S}(0)+A_{\mathcal{S}}(x)$ and $\mathcal{T}(x)=\mathcal{T}(0)+A_{\mathcal{T}}(x)$. Assume that $\mathcal{S}$ left commutes with $\mathcal{T}$, that is,

$$
\mathcal{S T} \subset \mathcal{T S}
$$

and that $\mathcal{T}$ is compact. Theorem 4.3 implies that the operators $A_{\mathcal{S}}$ and $A_{\mathcal{T}}$ commute with each other, that is,

$$
A_{\mathcal{S}} A_{\mathcal{T}}=A_{\mathcal{T}} A_{\mathcal{S}} .
$$

Since $A_{\mathcal{T}}$ is an operator part of a compact linear relation $\mathcal{T} \in \operatorname{LR}_{0}(X)$, Lemma 3.3 implies that $A_{\mathcal{T}}$ is compact. The commutativity in (5.1) implies (by Lomonosov's results) that the operator part $A_{\mathcal{S}}$ of the linear relation $\mathcal{S} \in \mathrm{LR}_{0}(\mathrm{X})$ has a nontrivial invariant subspace. The desired result then follows from Lemma 5.1.

\section{Acknowledgment}

I would like to thank the referee for his/her useful comments.

\section{References}

[1] N. Aronszajn, K. T. Smith, Invariant subspaces of completely continuous operators, Ann. Math., 60, (1954), 345-350. 1, 3,5

[2] C. Constantinescu, C*-Algebras, Volume 1: Banach spaces, North-Holland Mathematical Library, Amsterdam, (2001). 1

[3] R. Cross, Multivalued linear operators, Marcel Dekker Inc., New York, (1998). 1, 2, 2

[4] C. S. Kubrusly, An introduction to models and decompositions in operator theory, Birkhauser, Boston, (1997).

[5] E. Kreyszig, Introductory functional analysis with applications, John Willy \& Sons, New York, (1978). 3

[6] V. I. Lomonosov, Invariant subspaces for the family of operators which commute with a completely continuous operator, Funct. Anal. Appl., 7 (1973), 213-214. 1

[7] R. Meise, D. Vogt, Introduction to functional analysis, Oxford University press, New York, (1997). 1

[8] P. Saveliev, Lomonosov's invariant subspace theorem for multivalued linear operators, Proc. Amer. Math. Soc., 131 (2003), 825-834. 1, 2

[9] G. Wanjala, The invariant subspace problem for absolutely p-summing operators in Krein spaces, J. Inequal. Appl., 2012 (2012), 13 pages. 3

[10] G. Wanjala, Operator representation of sectorial linear relations and applications, J. Inequal. Appl., 2015 (2015), 16 pages. 